\title{
Roles of Paper Composition and Humidity on the Adhesion between Paper Sheet and Glass: A Molecular Dynamics Study
}

Hyunhang Park

Corning Precision Materials

Sung Hoon Lee ( $\nabla$ sunghoon.lee@corning.com )

Corning Precision Materials https://orcid.org/0000-0002-4320-4834

Elizabeth I. Morin

Corning Inc

Andrew C. Antony

Corning Inc

\section{Research Article}

Keywords: paper sheet, silica glass, molecular dynamics, adhesion, crystalline cellulose, xylan hemicellulose

Posted Date: February 12th, 2021

DOI: https://doi.org/10.21203/rs.3.rs-183769/v1

License: (c) (i) This work is licensed under a Creative Commons Attribution 4.0 International License. Read Full License 


\section{Roles of Paper Composition and Humidity on}

\section{the Adhesion between Paper Sheet and Glass:}

\section{A Molecular Dynamics Study}

Hyunhang Park ${ }^{*}, 1$, Sung Hoon Lee ${ }^{\dagger, 1}$, Elizabeth I. Morin ${ }^{2}$, Andrew C. Antony ${ }^{3}$

${ }^{1}$ Corning Technology Center Korea, Corning Precision Materials Co., Ltd., 212 Tangjeong-ro, Asan, Chungcheongnam-do 31454, Republic of Korea

${ }^{2}$ Science and Technology Division, Corning Incorporated, One Science Center Drive, Corning, New York 14831, United States

${ }^{3}$ Manufacturing, Technology, and Engineering Division, Corning Incorporated, One Science Center Drive, Corning, New York 14831, United States

E-mail: hyunhang.park@ corning.com, sunghoon.lee@ corning.com

(C) 2021 Corning Incorporated. All Rights Reserved.

*Primary Authors: Tel: +82-41-520-5674 


\begin{abstract}
Understanding adhesion behavior between paper materials and inorganic substrate is important to minimize surface contamination by paper fragments. In this work, we investigate adhesion mechanisms between paper sheet and glass in terms of molecular interaction. Molecular dynamics simulations are employed to calculate adhesion force between paper sheet and the silica glass surfaces. Pulling and sliding tests are simulated to find the effects of the paper compositions such as mannan, glucan, and glucuronoxylan and humidity on the adhesion. Simulation results reveal that crystalline cellulose film of mannan unit (uni-directional hydroxyl groups) shows higher adhesion than that of glucan unit (bidirectional hydroxyl groups). Also, addition of just a few glucuronoxylan (xylan) hemicellulose molecules on the cellulose film remarkably enhances adhesion force, because of carboxylic acid groups with strong polarity in xylan. In addition, introduction of humidity leads to a further increase of adhesion due to hydrogen bonds bridged by water molecules. The adhesion force is maximized around 10 $\mathrm{H}_{2} \mathrm{O} / \mathrm{nm}^{2}$ because as the humidity increases, hydrogen bond interactions are saturated, whereas the Van der Waals interactions decrease due to thicker water layer. It is discussed that consideration of the capillary force for paper may result in different adhesion response that reflects more realistic situation.
\end{abstract}

Keywords: paper sheet, silica glass, molecular dynamics, adhesion, crystalline cellulose, xylan hemicellulose 


\section{INTRODUCTION}

Packaging paper is used to protect underlying substrates during transportation and storage. To preserve substrate surface cleanliness, particularly for high tech applications whose surface quality is of great importance, it is ideal that the paper survive a wide range of humidity with minimal interaction with the substrate. Deposition of paper moieties onto the substrate surface during use and sticking, even at the micrometer-scale, may result in severe deterioration of surface quality. In this regard, the fundamental solution for those issues should start with an understanding of adhesion behavior at the interface from the molecular scale. Furthermore, it is expected that this understanding can contribute to chemical optimization of paper components or control of ambient conditions for minimization of the adhesion strength.

In general, interfacial adhesion is dependent on many factors including the nature of the interfacial interaction, mechanical locking, chemical reaction, and interdiffusion.(Awaja 2009; Lacomb 2006) In ambient conditions, it is generally accepted that a major factor in the adhesion between paper material and relatively hard substrates, such as glasses or metals, is a non-bonded type of interaction which includes Van der Waals and/or Coulomb interactions.(Min 2016; Miwa 1993; Yarovsky 1997) Thus, we limit our focus on the non-bonded interactions between the paper and glass in the present work. Nevertheless, under a normal laboratory type of environment, many other kinds of interactions due to relative humidity and organic contaminant levels are added. These factors make the fundamental understanding of relevant adhesion mechanisms all the more difficult, but equally critical. 
There have been hundreds of studies on the mechanical properties of cellulose, hemicellulose and lignin in the plant cell wall as raw materials for the paper.(Charlier 2012; Gibson 2012; Mazeau 2012; Molnar 2018; Paavilainen 2012; Zhang 2015) However, studies of adhesion phenomena of the paper material highly modified from the living cell structure through the pulping process have been rare. As a relevant experiment, Goswami et al. discuss the adhesion between cellulose nanocrystal (CNC) and glass fiber/epoxy composite.(Goswami 2019) Authors show that the CNC enhances the interfacial shear strength in glass fiber/epoxy composites due to interphase toughening with interpenetrating network formation. However, they did not consider a paper sheet which is a complex entanglement of cellulose microfibrils, nor the pure glass structure. There have been several computational works that focused on the fundamental aspects of adhesion between the silica glass and various organic polymer systems, from a single molecule all the way to complex thin film networks.(Goyal 2016; Min 2016; Min 2018) However, the systematic study of adhesion behavior between the paper material and the glass substrate is still lacking.

In this regard, we aim to understand the mechanism of adhesion behavior between the paper and fused glassy silica via molecular dynamics simulations. In order to elucidate the effect of main components of the paper on the adhesion, crystalline cellulose film and hemicellulose-cellulose composite are generated, and the interfaces with the silica glass are prepared for the adhesion calculation. Steered molecular dynamics (SMD) technique is employed to mimic experimental pulling and sliding tests on the interfaces, and failure behavior of the film is monitored. Based on the temporal change of free energy during test, the resultant adhesion forces and energies are calculated. Adhesion trends of cellulose and cellulose-hemicellulose composite are (C) 2021 Corning Incorporated. All Rights Reserved. 
compared, and underlying mechanism is suggested. Finally, the effects of relative humidity on the adhesion are investigated considering it as a major environmental factor. The amount of interfacial water content is controlled, and the resultant trends of adhesion are analyzed for both kinds of interfaces.

\section{COMPUTATIONAL DETAILS}

\section{Materials}

The interface in this work is composed of paper film and a glass substrate. The basic molecular building block of paper is cellulose, which is a linear chain made of Dglucose unit and connected by $\beta$-1, 4-linked glycosidic bonds. The chemical formula of the cellulose is $\left(\mathrm{C}_{6} \mathrm{H}_{10} \mathrm{O}_{5}\right)_{n}$. Specifically, cellulose $\mathrm{I} \beta$ structure was chosen as a dominant crystalline form of cellulose in plants and woods.(Nishiyama 2009; Zugenmaier 2008) Hemi-cellulose, the second major ingredient of the paper, contains sugar monomers such as glucose, xylose, or mannose and so on; thus, its structure is different from cellulose in the sense that it has a random and amorphous structure. We consider Glucuronoxylan (xylan) from hardwood, which consists of $\beta$-1, 4-linked Dxylopyranose backbone with 4-O-Me- $\alpha-\mathrm{D}-$ Glucuronic acid residues at $\mathrm{O}-2$ of one of every 6 xylans as suggested in Zhang et al.(Zhang 2015) Figure 1(a) and 1(b) show the chemical structures of cellulose I $\beta$ and the xylan molecule. For the glass substrate, amorphous silica structure is considered as an archetype of the glass whose surface is 
fully covered by hydroxyl groups with the surface density of $4.6 \mathrm{OH} / \mathrm{nm}^{2}$.(Zuravlev 2000)

\section{Construction of interfaces}

Due to a large amount of hydroxyl groups in the D-glucose unit, repeat units of cellulose I $\beta$ tend to be polymerized to a linear chain. In addition, the linear chains try to make strong intra- and inter-molecular hydrogen bonds with each other and finally generate a monoclinic crystalline structure. In the case of living plants, the crystalline cellulose plays a role as skeleton of the plant cell with hemicellulose and lignin components.(Gibson 2012) However, typical paper manufacturing process under high temperature and pressure destroys most of the crystallinity of the cellulose structure, leaving some fragments of fiber shape. Those entangled mixtures of the cellulose fragments called microfibril bundles are the main feature of the paper material of our interest. It would be reasonable to construct a flat film of the cellulose chains in the MD simulation, because microfibrils are almost flat in the axial direction at the nanometer scale; furthermore, we are focusing on the surface part of the microfibril which is especially responsible for the adhesion strength on the glass surface.

For the construction of cellulose film structure, one monolayer is formed by 7 cellulose chains attached to each other in parallel along the $y$ direction, and 4 monolayers are piled up in the $z$ direction with a height of $7 \mathrm{~nm}$. For the silica substrate, the protocol suggested by Lee et al. are used to generate the amorphous silica substrate with the lateral size of $6 \times 12 \mathrm{~nm}^{2}$.(Lee 2017) For the building of cellulose- 
silica interface, the cellulose film is initially placed at a $1 \mathrm{~nm}$ height above the silica substrate. The periodic boundary condition is applied in $x$ and $y$ directions while $z$ direction remains fixed to prevent any neighboring interactions between the top and bottom of the simulation box. Through a thermal annealing protocol up to $600 \mathrm{~K}$, the cellulose film is uniformly adsorbed to the silica to form a realistic cellulose-silica interface. The perspective view of the relaxed interface is shown in figure 1(c).

We also consider xylan molecules which are adsorbed on the surface of the cellulose film. However, information of their precise configurations is also needed for the construction of the cellulose-xylan composite film because conformational degrees of freedom of xylan is very high due to its high flexibility. Recently, it has been reported that backbones of the xylan molecules are adsorbed as maintaining fully stretched configuration.(Mazeau 2012) Thus, we introduce xylan molecules of a stretched shape just above the silica surfaces so that they can be adsorbed while maintaining the stretched configuration. We consider two cases of this interface where 2 and 6 xylan molecules are adsorbed on the cellulose film. Figure 1(d) displays a top-down view of the cellulose surface with 6 xylans adsorbed.

To examine the impact of humidity on the paper-silica adhesion, water molecules are introduced to the interface between paper and silica. For interface building, a layer consisting of water molecules with specific density is initially placed between the cellulose-xylan composite film and the silica substrate. Then the whole system is relaxed at room temperature for $3 \mathrm{~ns}$, forming the composite-water-silica multilayer system. Since main adsorption sites of the molecular water on the silica surface are thought to be surface silanol groups,(Stolper 1982; Zuravlev 2000) the amount of water molecules introduced in this simulation starts from $5 \mathrm{H}_{2} \mathrm{O} / \mathrm{nm}^{2}$ which is similar to (C) 2021 Corning Incorporated. All Rights Reserved. 
hydroxyl density of $4.6 \mathrm{OH} / \mathrm{nm}^{2}$, and three more cases: 10,15 , and $20 \mathrm{H}_{2} \mathrm{O} / \mathrm{nm}^{2}$. For this range of water densities, the thickness of water layers relaxed between the composite and silica were calculated to be around $0.4,0.7,0.9$, and $1.1 \mathrm{~nm}$, respectively. As a relevant experiment, Verdaguer et al. measured the thickness of water layers on $\mathrm{SiO}_{2}$ substrate, and reported that thicknesses of $0.6 \mathrm{~nm}$ to $1.3 \mathrm{~nm}$ correspond to relative humidity of $15 \%$ and $75 \%$, respectively.(Verdaguer 2007)

All of the initial structures shown above except for silica substrate were constructed with the configuration biased Monte Carlo technique (Amorphous Cell module from Materials Studio 8.0 Package).(Materials Studio, Software for Technical Computation 2006) Thermal annealing and relaxation procedures were performed by using LAMMPS simulation package(Plimpton 1995) with the Interface-PCFF forcefield.(Heinz 2013) NVT ensembles with Nosé-Hoover thermostat and barostat were used throughout all simulations and the time step was set to be $1 \mathrm{fs}$. The cutoff for van der Waals interactions is $12 \AA$, and the Particle-Particle Particle-Mesh (PPPM) solver is used for the summation of long-range Coulomb interactions with the precision of $10^{-4}$. Lennard-Jones forcefield was used for the interaction between the water molecules whose charges are assigned using the simple point charge (SPC) model.(Berendsen 1987) For the interactions between water and other layers, the same Interface-PCFF forcefield is applied.

Simulation technique for adhesion calculation

To calculate adhesion properties and monitor the failure behavior at the paper-silica interface, we use steered molecular dynamics (SMD) technique to mimic the () 2021 Corning Incorporated. All Rights Reserved. 
experimental pulling and sliding tests. SMD in the mode of pulling test has been utilized to estimate the interfacial adhesion and analyze conformational changes of the polymer systems(Goyal 2016; Lee 2017; Min 2016; Min 2018) and biomaterials,(Chabria Dec. 2010; Davis May 2009) and it was also reported that SMD may be successfully applied to mimic peeling and sliding test.(Min 2017) In this technique, a fictitious atom is connected to the center of mass (COM) of the system of interest by a virtual spring with a spring constant $k$, and the atom pulls it along a specific direction from the silica surface with a constant velocity $v$. During the process, the total force and the potential energy are calculated as following equations:

$$
\begin{aligned}
& U_{\text {spring }}=\frac{1}{2} k\left[v t-\left(R(t)-R_{0}\right) \cdot n\right]^{2} \\
& F_{\text {spring }}=-\nabla U_{\text {spring }}
\end{aligned}
$$

where $R(t)$ is the current position of the COM of the system, $R_{0}$ is the initial center of mass of the system, and $n$ is a unit vector along the direction in which the spring is pulled. Then the total work done is calculated as:

$$
W=\int_{r=R_{0}}^{r=R_{f}} \nabla U_{\text {spring }} \mathrm{d} \vec{r}
$$

where $R_{f}$ is the final position of the COM of the system. It is well known that the ensemble average of total work done can be regarded as the potential of mean force (PMF) using Jarzynski equality(Jarzynski 1997; Park 2004) : 


$$
\langle\exp (-\beta W)\rangle_{\text {ensemble }}=\exp \left(-\beta U_{P M F}\right)
$$

where $\beta=\frac{1}{k_{B} T}, k_{B}$ is the Boltzmann contant, and $T$ is the temperature. The applied pulling velocity for SMD simulation is $0.75 \mathrm{~m} / \mathrm{s}$, which has been validated as slow enough to calculate the adhesion precisely in the previous reports.(Goyal 2016; Lee 2017)

Throughout the present work, pulling and sliding tests indicate the situation where the atom is pulled in the parallel and normal direction to the interface, respectively. Figure 2 illustrates basic scheme of pulling and sliding tests and the evolution of PMF and relevant quantities which are calculable during the tests. In the case of pulling test, PMF is saturated when the paper film is completely detached from the silica substrate. The pulling distance, saturated PMF value, and maximum height of the pulling force are denoted as $D_{\text {det }}, E_{a d h}$, and $F_{\text {max }}$, respectively. On the other hand, since PMF just continuously increases without saturation during the sliding test, only the value of sliding force is considered as a meaningful adhesion quantity. The sliding force value is averaged over the sliding distance of $5 \AA$ after the force is saturated and is denoted as $F_{\text {avg }}$.

\section{RESULTS AND DISCUSSIONS}

Cellulose-glass interface 


\section{Adhesion at cellulose-glass interface: pulling test}

We start our study from the adhesion behavior between cellulose film and silica substrate. To clarify the effect of molecular interaction on the adhesion as well as estimate adhesion level, two kinds of cellulose films whose D-glucose parts are glucan and mannan glucose unit are compared. As shown in figure 3, the difference in molecular structures of glucan and mannan units can be described as follows: in the glucan unit, there is a set of trans hydroxyl groups (i.e. two hydroxyl groups are on opposite sides of the ring), whereas in the mannan unit, there is a set of cis hydroxyl groups (i.e. two hydroxyl groups are on the same side of the ring). More specifically, cis hydroxyl groups can have up-side or down-side directions where the cellulose film is constructed, and in this simulation only cis orientation with down-side direction was considered.

Figure 4 exhibits a typical evolution of pulling force and PMF curves as a function of pulling distance for glucan-silica and mannan-silica interfaces. One can immediately find that both $E_{a d h}$ and $F_{\max }$ for the mannan-silica interface are higher than those for the glucan-silica interface. Adhesion values averaged over 5 samples show the differences in the level of $E_{a d h}$ and $F_{\max }$ are $40 \%$ and $15 \%$, respectively. It is easily understood that the structural difference between the glucan and mannan units in the cellulose film is a critical factor for their difference in adhesion. In other words, with both hydroxyl groups on the same down-side of the ring in the cis orientation in mannan, more hydrogen bonds with the substrate can be simultaneously formed than glucan case. As a result, there should be more hydrogen bonding interactions between cellulose film and the silica substrate, which leads to stronger adhesion strength. We 
also note that if direction of the hydroxyl groups in mannan were up-side of the ring at the bottom layer, then the adhesion would be smaller than glucan case.

\section{Adhesion at cellulose-glass interface: sliding test}

To mimic the rubbing motion between the paper sheet and the silica, the sliding test is also performed at the cellulose-silica interface. Figure 5 shows the temporal evolution of the sliding force applied along the $x$ and $y$ directions. It is observed that sliding forces result in oscillatory curves, which reflect the crystallinity of the cellulose film. Also, sliding force in the $y$ direction is higher than the $x$ direction because the chain is polymerized along the $y$ direction whereas weakly combined by intermolecular interaction along the $x$ direction.

Averaged over the $x$ and $y$ directions, the resultant $F_{\text {avg }}$ for glucan- and mannansilica interfaces are found to be 17.2 and $64.7 \mathrm{kcal} / \mathrm{mol} / \AA$, respectively. It is not surprising that $F_{\text {avg }}$ for manna-silica interface should be higher than that for glucansilica interface considering the results of our pulling test. However, it is remarkable that the difference in $F_{\text {avg }}$ between the two interfaces is more than three times. This result indicates that angular and torsional interactions between the surface hydroxyl groups of the cellulose and silica provide more contribution to $F_{\text {avg }}$ than the pulling test. Since the sliding process increases the probability of contacts between the functional groups of cellulose and glass, molecular interactions between them are also expected to increase. Overall, by comparing the pulling and sliding simulations, we can conclude that the surface density of the polar functional groups such as hydroxyl groups significantly affect the adhesion at the cellulose-silica interface. 


\section{Addition of hemicellulose}

Figure 6 compares adhesion forces between the cellulose only case and the cellulosexylan composite case for pulling and sliding tests. Figure 6(a) shows that $F_{\text {max }}$ of the cellulose-xylan composite case is much lower than the cellulose only case during the pulling test. On the other hand, in the sliding test, $F_{a v g}$ of the cellulose-xylan composite case is much higher than the cellulose only case as shown in Figure 6(b).

This apparent difference in the trend can be understood from analyzing the detailed geometry of the interface. When thermal annealing is performed to generate the interface between cellulose-xylan composite and the silica substrate, the lower part of the surface at which xylan molecules are adsorbed uniformly tries to cover the silica. However, since the cellulose film is relatively rigid along the $y$ direction (the direction of chain polymerization), some cavities at the nanometer scale are created around the adsorbed xylan molecules along that direction. These nanocavities weaken the strength of inter-molecular interactions at the interface, which leads to a decrease of $F_{\max }$ when compared to the cellulose only case where the whole film is uniformly adsorbed to the silica. In the sliding test, on the contrary, it is noteworthy that the cellulose-xylan composite and silica keep their molecular interactions during the entire sliding process, and thus the interface has more chances to form strong interactions between polar functional groups. Indeed, it was observed that the xylan molecules originally adsorbed to the composite move with the silica substrate, in the opposite direction of the cellulose part of the composite film. This implies that the carboxylic 
acid groups in the xylan molecule may easily find proper binding sites to form very strong hydrogen bonds with the hydroxyl groups on the silica surface during sliding test. It also suggests that a few polar functional groups may play a role of critical factor for high sliding force as exemplified in figure 6(b).

It is also remarkable that for both pulling and sliding tests, one can observe higher adhesion for 2 xylan added case than that for 6 xylan added case. This can be also explained by imagining the concept of nanocavity at the interface. Since 2 xylan case has more spacing between the xylans than 6 xylan case, more part of the cellulose film will be adhered to the silica substrate. Then more nanocavities will be collapsed for 2 xylan cases during thermal annealing, which leads to more cellulose-silica interactions and finally higher adhesion.

Effect of relative humidity

\section{Adhesion at a low humidity}

Since paper material is highly hydrophilic due to noncrystalline hemicellulose structure and the porosity between loosely entangled microfibrils,(Kargarzadeh 2017) one can imagine that the adhesion level should increase even at low humidities. To first check the effect of humidity on the paper-glass adhesion, we calculated adhesion under humid condition with $5 \mathrm{H}_{2} \mathrm{O} / \mathrm{nm}^{2}$ to compare with the dry condition. The snapshots in figure 7 show how including water at the interface is different from the interface without water. The cellulose-xylan composite and silica substrate in the interface are denoted as small dots, and only $\mathrm{COOH}$ in xylan and water molecules are displayed as circles for clarity. At the low humidity interface, water molecules occupy nanocavities (C) 2021 Corning Incorporated. All Rights Reserved. 
which caused low adhesion of the interface in the dry case. The water molecules also diffuse into both the cellulose chains and silica surface to form small clusters bridging polar functional groups such as carboxylic acid in the xylan or hydroxyl groups in cellulose and silica surface. Therefore, one can imagine that the water molecules may reinforce interactions between the composite and the silica by playing a role of 'glue' to adhere cellulose-xylan composite and silica.

Indeed, figure 8 clearly shows that the adhesion levels for both pulling and sliding tests are significantly increased once the water molecules are introduced. Figure 8(a) shows that in the pulling test, the contribution of water molecules to the adhesion is large enough to overcome previous low adhesion observed for the cellulose-xylan composite film. Also, the adhesion level in the sliding test strikingly increases more than three times as shown in Figure 8(c). It is thought that the sliding action evenly distributes the water molecules over the whole interface to increase adhesion further. To conclude, even low humidity conditions may significantly enhance adhesion strength between paper and glass by creating additional hydrogen bonded bridges between the surface hydroxyl groups of the silica and polar functional groups of the cellulose and xylan.

\section{Trend of adhesion for humidity increase}

Next, we consider more humid conditions up to $20 \mathrm{H}_{2} \mathrm{O} / \mathrm{nm}^{2}$ to calculate the change of paper-glass adhesion. The resultant trend of adhesion is displayed in figure 9. Interestingly, the trend approaches a maximum around $10 \mathrm{H}_{2} \mathrm{O} / \mathrm{nm}^{2}$ for both pulling and sliding tests. The trend of increasing adhesion until $10 \mathrm{H}_{2} \mathrm{O} / \mathrm{nm}^{2}$ is easily understood considering the contribution of additional hydrogen bonds by more water (c) 2021 Corning Incorporated. All Rights Reserved. 
molecules. However, it is notable that water molecules can form a well-defined layer once they cover the whole glass surface as indicated by figure 10(a) where water density is $20 \mathrm{H}_{2} \mathrm{O} / \mathrm{nm}^{2}$. Figure 10(b) shows that the center-of-mass distance between the cellulose-xylan composite and the silica continuously increases with increasing humidity. This continuous increase of interfacial distance due to the thicker water layer leads to a reduction of the molecular interactions at the interface, and finally lower adhesion. It is expected that the tendency of adhesion increase by additional water molecules is saturated at some humidity level, whereas the tendency of adhesion reduction due to increasing separation distance will be maintained and continuously decrease. Since the former tendency will dominate the latter from 0 to $10 \mathrm{H}_{2} \mathrm{O} / \mathrm{nm}^{2}$, the adhesion energy and forces increase. Once the additional adhesion due to water molecules is saturated at $10 \mathrm{H}_{2} \mathrm{O} / \mathrm{nm}^{2}$, the decreasing strength due to increasing separation distance dominates which causes declining adhesion energy and forces after a RH level of $10 \mathrm{H}_{2} \mathrm{O} / \mathrm{nm}^{2}$. we can conclude that the adhesion curves in figure 9 draw a maximum at that point.

\section{Effect of capillary force}

At the macroscopic scale, it is natural to expect some capillary action at the interface, especially for the high humidity scenario. However, in the current simulation the water meniscus which is qualitative evidence of capillary action was not observed even for $20 \mathrm{H}_{2} \mathrm{O} / \mathrm{nm}^{2}$. Possible reasons for that would be twofold: first, paper fragments causing the surface contamination may include larger size fractions that cannot be modeled on the nanoscale of a MD simulation. Second, the amount of water which is supposed to be within the interface may not be enough to induce capillary behavior. Values of water (C) 2021 Corning Incorporated. All Rights Reserved. 
densities used in this study have been estimated from the hydroxyl density of 4.6 $\mathrm{OH} / \mathrm{nm}^{2}$ on the bare silica surface exposed to ambient temperature and pressure. Since the paper itself can also absorb water molecules which will be distributed near the surface, there might be more interfacial water than the bare glass surface when the paper fragment is attached to it. This factor can be tested in the current model set up.

In this regard, we additionally consider two cases of water number density as 40 and $80 \mathrm{H}_{2} \mathrm{O} / \mathrm{nm}^{2}$ to determine the trend of corresponding adhesion strengths. Figure 11 displays the results of adhesion calculation as a function of humidity. Both trends of $E_{a d h}$ and $F_{\max }$ clearly show that the change in adhesion as a function of humidity (i.e., the slope of the curve) diminishes as water number density increases. Comparison with the force curve in figure 12 provides a clue for understanding this phenomenon: as the amount of water is increased, the height of maximum point of force curve becomes lower, whereas the tail of the curve at large pulling distance increases. It should be noticed the tail part represents the water interaction which resists detachment of the two layers. Therefore, one can conjecture that the capillary force does contribute to the adhesion strength to some extent and we expect that the adhesion may finally increase for even more amounts of interfacial water.

\section{CONCLUSIONS}

We investigated adhesion phenomena between paper and silica glass as an example of heterogeneous organic-inorganic interface. To understand the mechanism of 
molecular interactions in the order of increasing complexity, we adopted MD simulations to prepare two kinds of paper compositions to interface with the glass: pure cellulose film and a cellulose-xylan composite film. Corresponding interfaces were constructed, and relevant adhesion properties such as adhesion energy, maximum sliding force, and average sliding force were calculated by pulling and sliding tests using the SMD technique. It was found that hydrogen bonds formed by abundant hydroxyl groups in the cellulose film play a critical role in forming strong adhesion at the cellulose-silica interface. Furthermore, the interface of cellulose-xylan composite and silica showed that even a small number of polar functional groups in xylan molecules significantly increases the adhesion strength. When comparing adhesion between two kinds of interfaces, adhesion force for the sliding test led to increased adhesion compared to the pulling test, which implies that rubbing motion of the paper on glass can induce significant contamination of the glass surface by paper fragments.

The effect of humidity on the adhesion of paper-silica interfaces was also intensively studied. Various amount of water molecules in the range of 0 to $80 \mathrm{H}_{2} \mathrm{O} / \mathrm{nm}^{2}$ were introduced to the paper-silica interface, and the adhesion mechanism was analyzed. Consistent with the dry condition, only a low humidity significantly enhanced adhesion between cellulose-xylan composite and silica, which is responsible for another set of hydrogen bonding provided by interfacial water molecules. Interestingly, adhesion level reached a maximum point in the range of 0 to $20 \mathrm{H}_{2} \mathrm{O} / \mathrm{nm}^{2}$. We conclude that reduction of the interfacial interaction by thickening of the water layer finally leads to a decrease of adhesion at humidity levels upward of $10 \mathrm{H}_{2} \mathrm{O} / \mathrm{nm}^{2}$. Even though capillary action was not observed in the current scale of MD simulation, the possibility of adhesion increase was confirmed with increasing water content. 


\section{DECLARATIONS}

Acknowledgement

We would like to thank Dr. Gabriel Agnello, Dr. Aravind Rammohan, and Dr. Hong Yoon for supporting our work.

Funding

The authors declare no competing financial interest.

Compliance with ethical standards

Conflict of interest

The authors declare that they have no conflict of interest.

\section{AUTHOR INFORMATION}

Corresponding Author

${ }^{\dagger}$ Sung Hoon Lee

E-mail address: sunghoon.lee@ corning.com; Tel +82-41-520-5691

ORCID: 0000-0002-4320-4834 

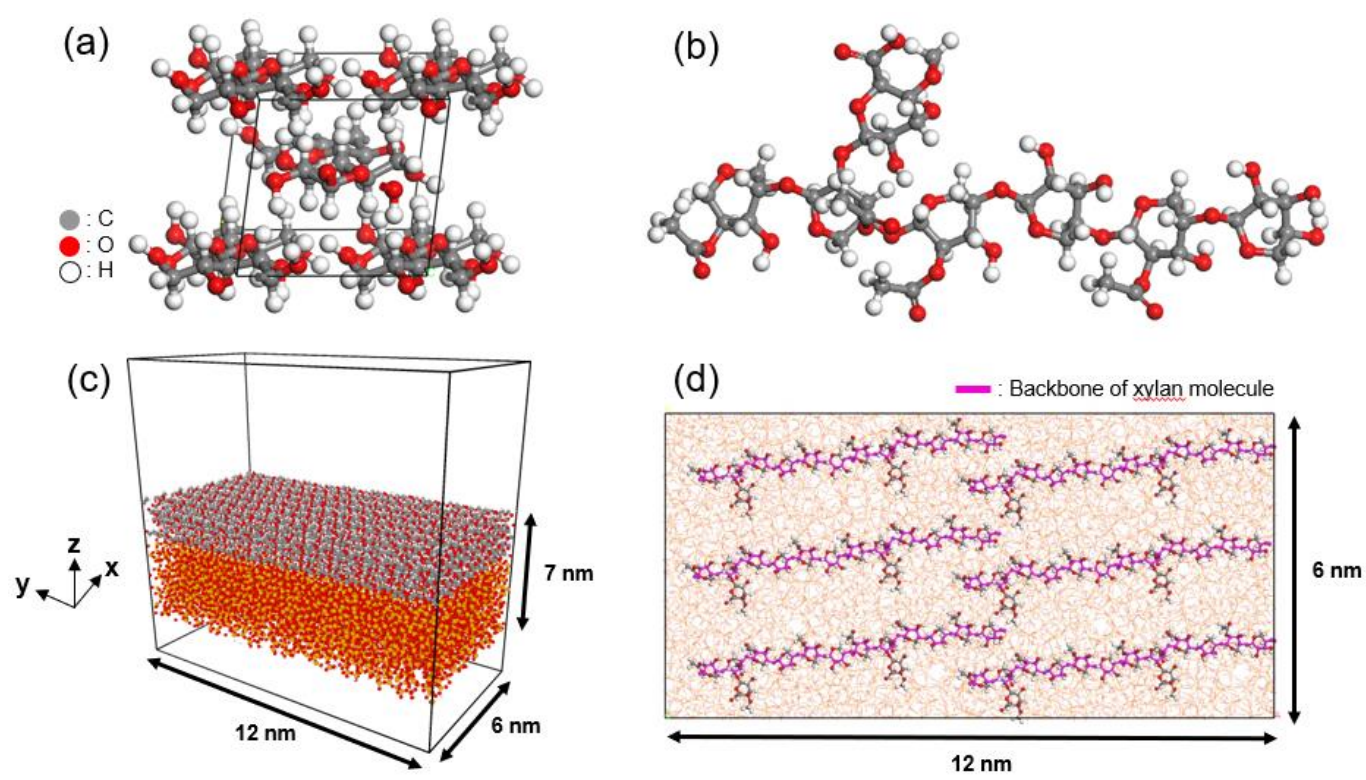

Figure 1. (a) Monoclinic crystalline structure of cellulose-I $\beta$ (b) Structure of Glucuronoxylan (xylan) hemicellulose molecule (c) Perspective view of the cellulose-silica interface after relaxation procedure (d) Bottom view of stretched configuration of 6 xylan molecules under the surface of cellulose film 


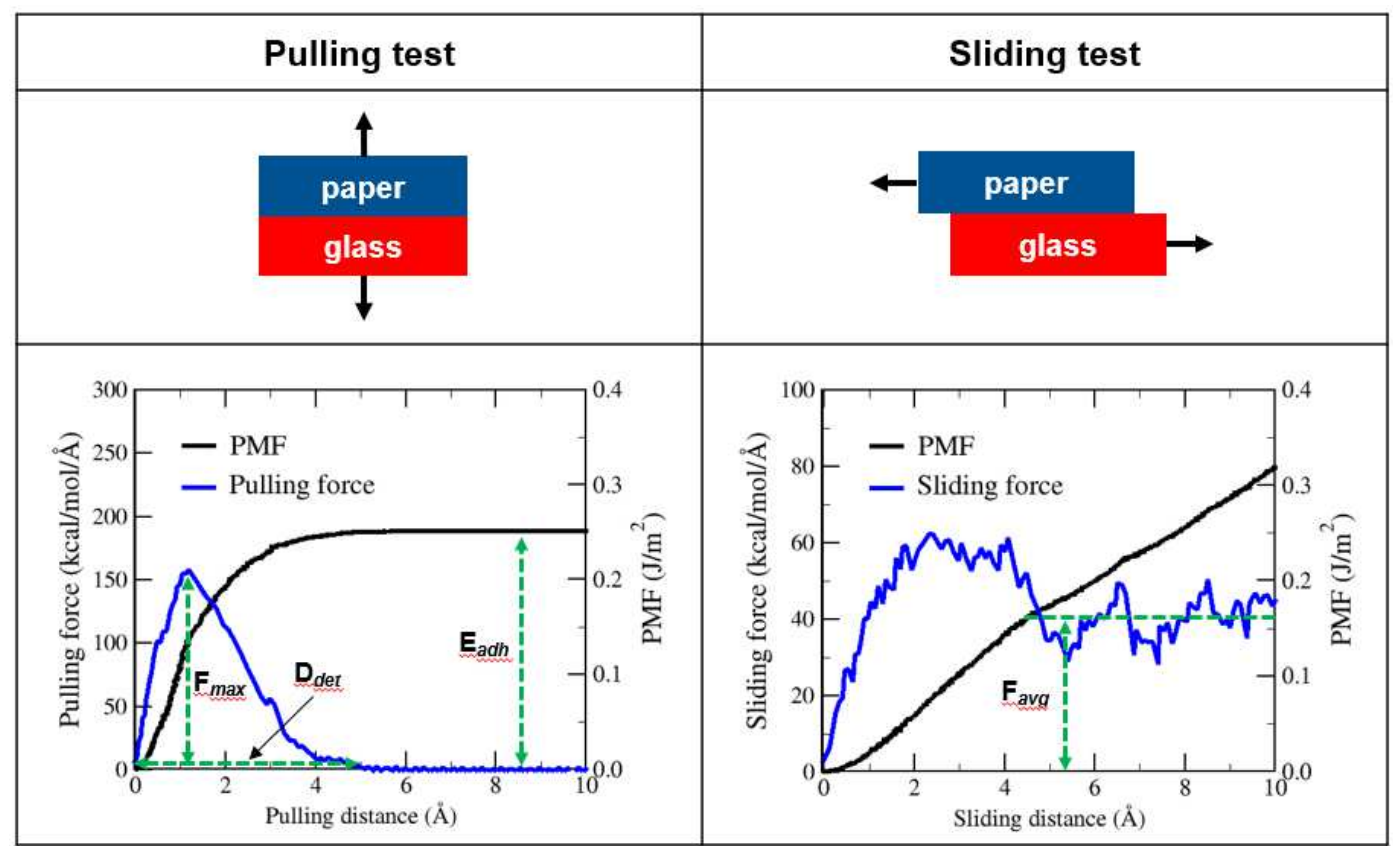

Figure 2. Comparison of pulling and sliding tests. Middle column shows basic scheme of tests, and bottom column describes typical evolution of PMF and forces and extractable adhesion properties 

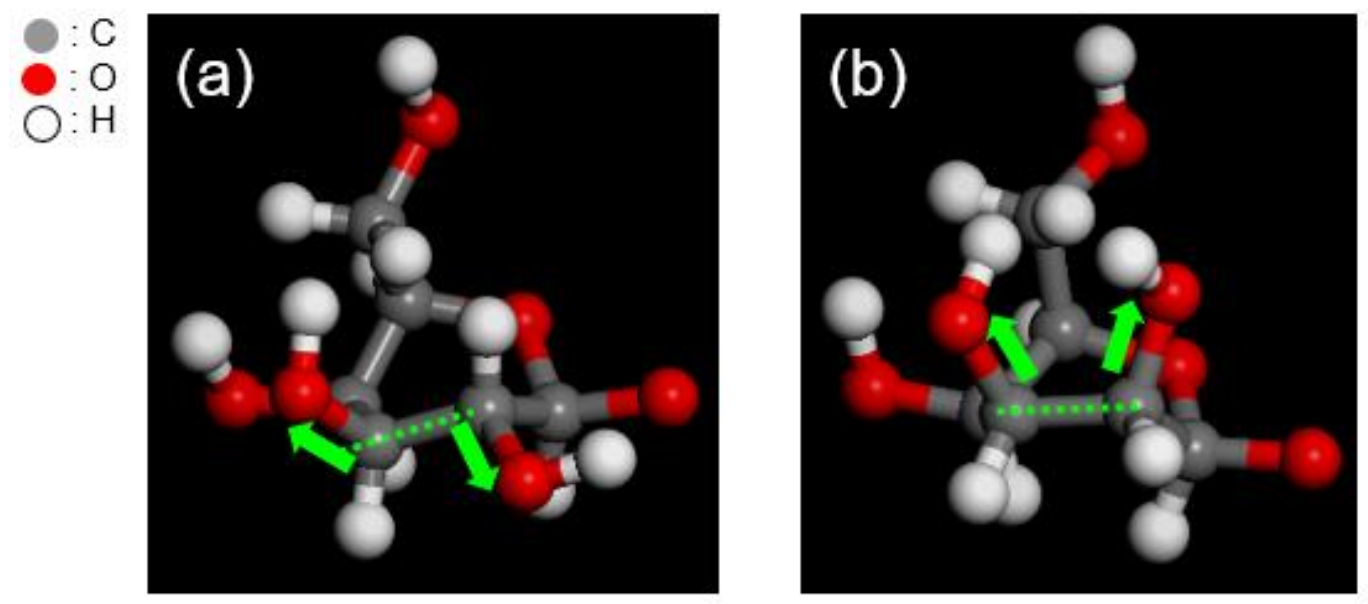

Figure 3. Chemical structures of glucose repeat units for (a) Glucan and (b) Mannan. Differences in the direction of hydroxyl groups are emphasized by green arrows. 

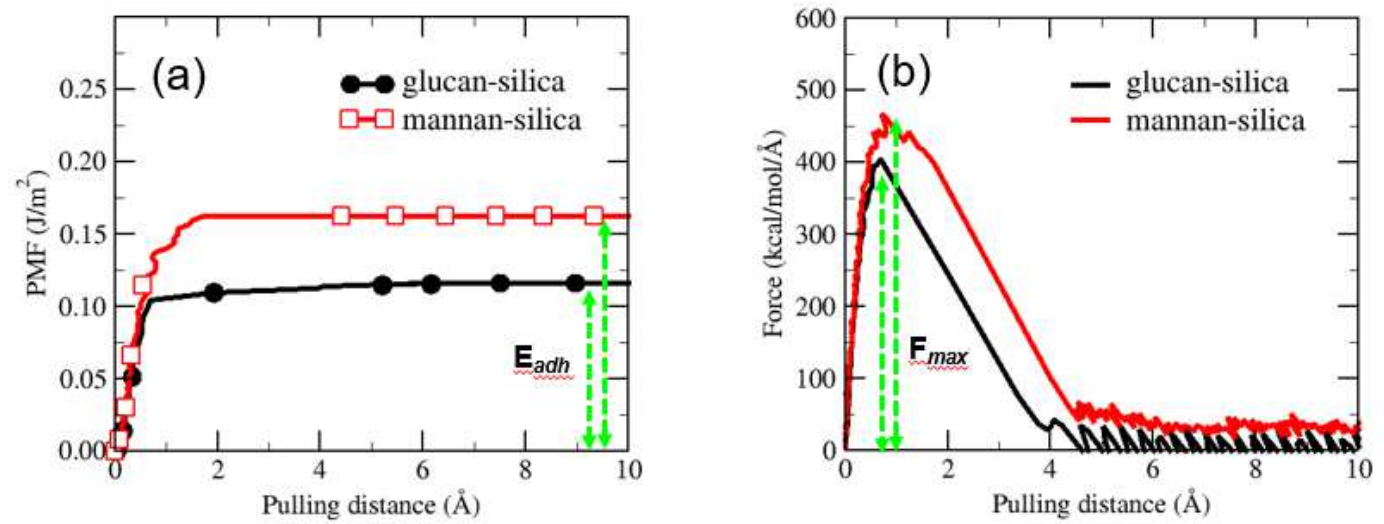

Figure 4. Comparison of adhesion properties between glucan- and mannan-silica interfaces during pulling test: (a) $E_{a d h}$ and (b) $F_{\max }$ 

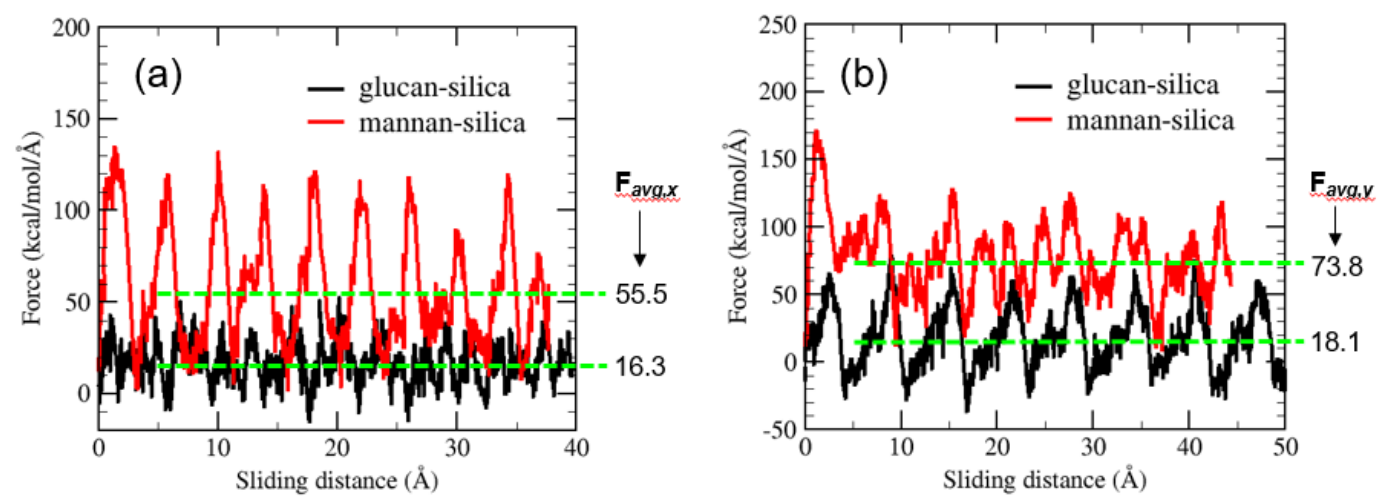

Figure 5. Comparison of sliding forces between glucan- and mannan-silica interfaces during sliding test along (a) $\mathrm{x}$ and (b) y directions. $F_{\text {avg }}$ values were averaged over the sliding distance from $5 \AA$ 

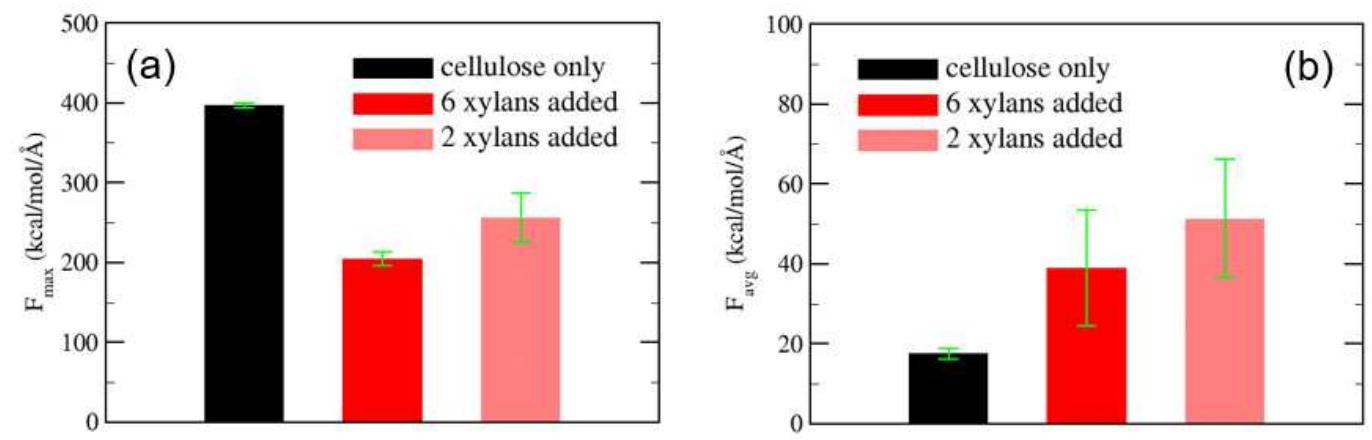

Figure 6. Comparison of adhesion forces between cellulose only case and cellulose-xylan composite case for (a) pulling and (b) sliding tests 

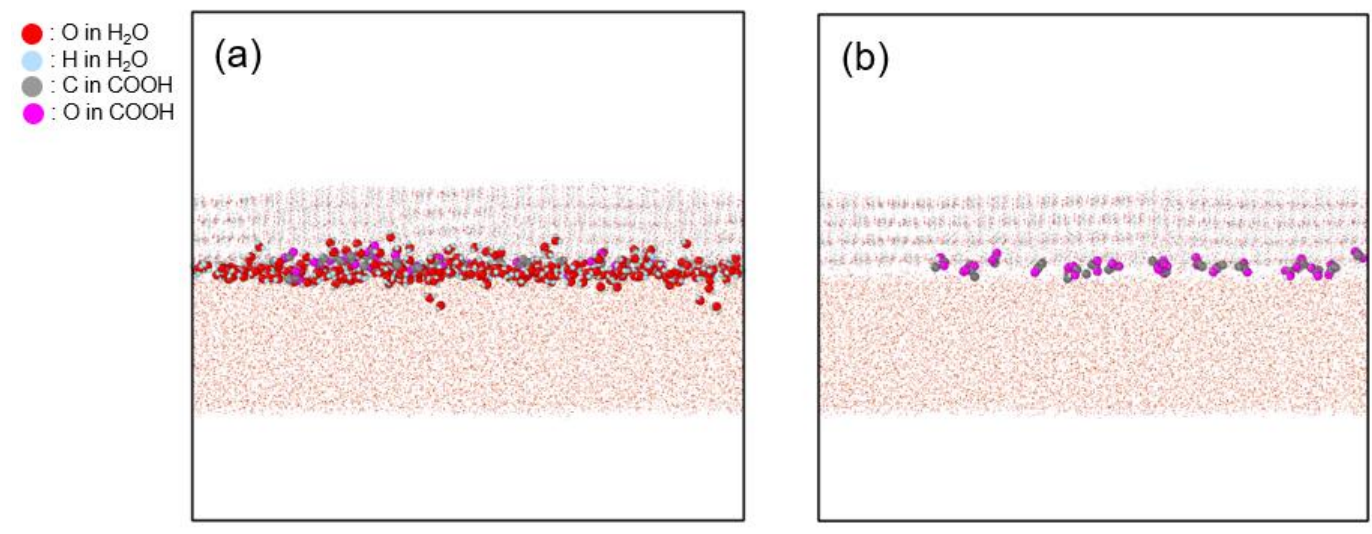

Figure 7. Snapshots of interface between cellulose-xylan composite and silica substrate under (a) 5 $\mathrm{H}_{2} \mathrm{O} / \mathrm{nm}^{2}$ and (b) dried condition. Only water molecules and $\mathrm{COOH}$ groups in xylan were emphasized with circles 

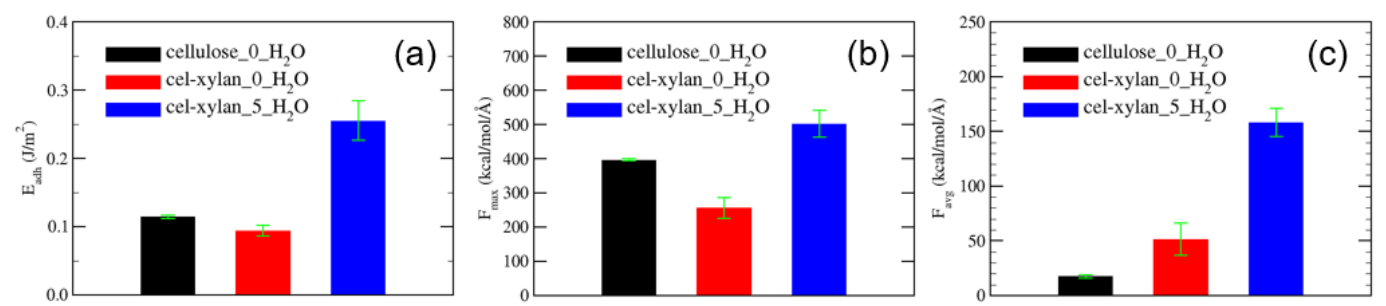

Figure 8. Adhesion properties of cellulose-xylan composite and silica under humid and dried conditions: (a) $E_{a d h}$, (b) $F_{\text {max }}$, and (c) $F_{\text {avg }}$ 

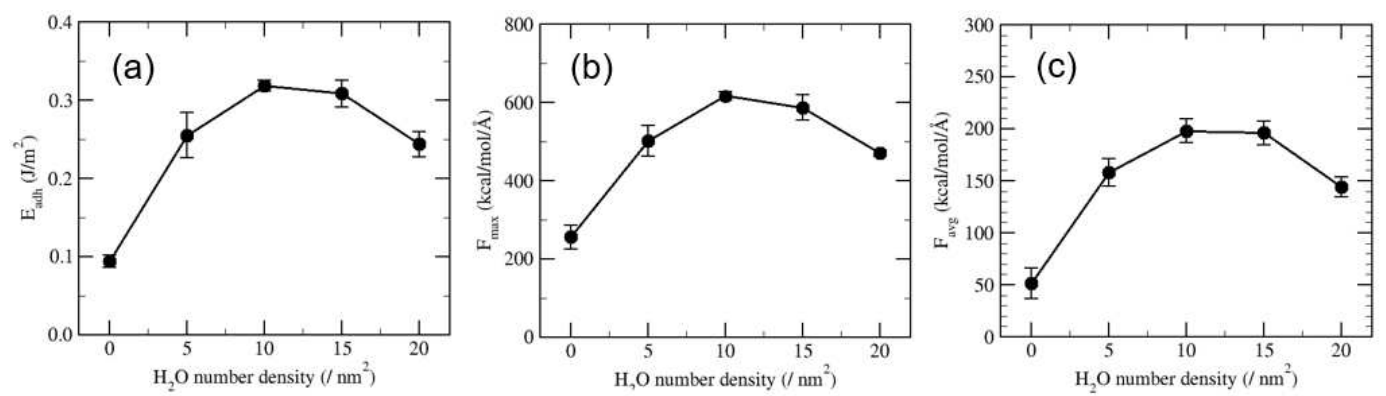

Figure 9. Trend of adhesion properties as a functions of $\mathrm{H}_{2} \mathrm{O}$ number density: (a) $E_{a d h}$, (b) $F_{\text {max }}$, and (c) $F_{\text {avg }}$ 

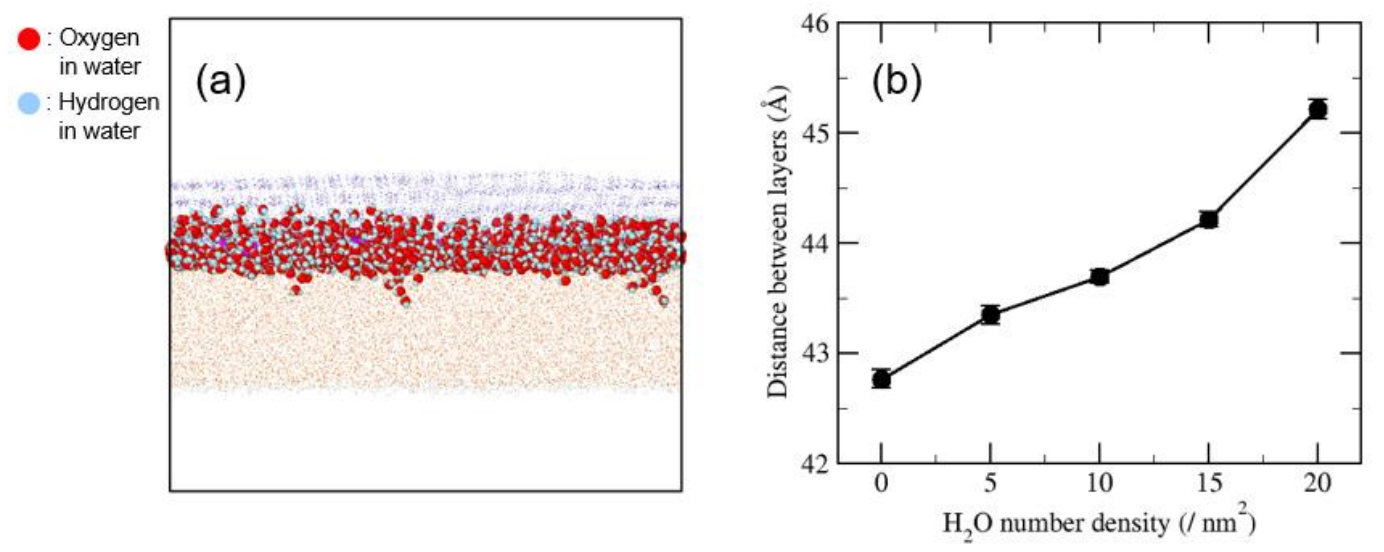

Figure 10. (a) water layer between cellulose film and silica surface at $20 \mathrm{H}_{2} \mathrm{O} / \mathrm{nm}^{2}$ (b) Distance between cellulose and silica increases with increasing humidity, which implies molecular interactions between the layers are reduced 

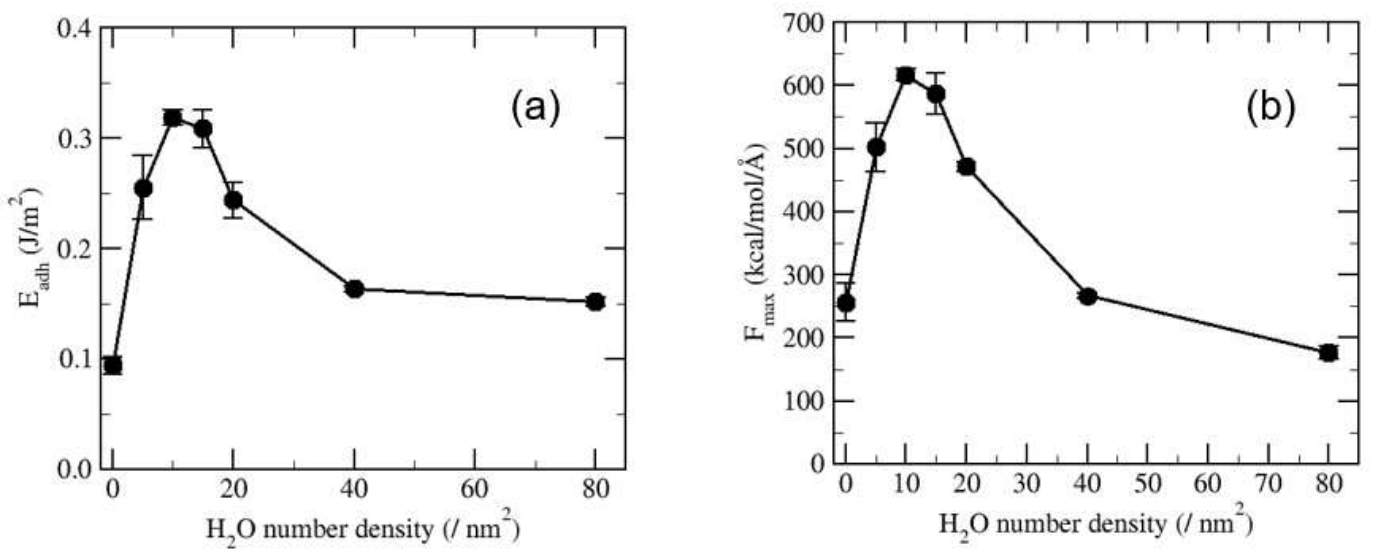

Figure 11. Curves of (a) $E_{a d h}$ and (b) $F_{\max }$ for paper-glass interface extended range of $\mathrm{H}_{2} \mathrm{O}$ number density up to $80 \mathrm{H}_{2} \mathrm{O} / \mathrm{nm}^{2}$ 


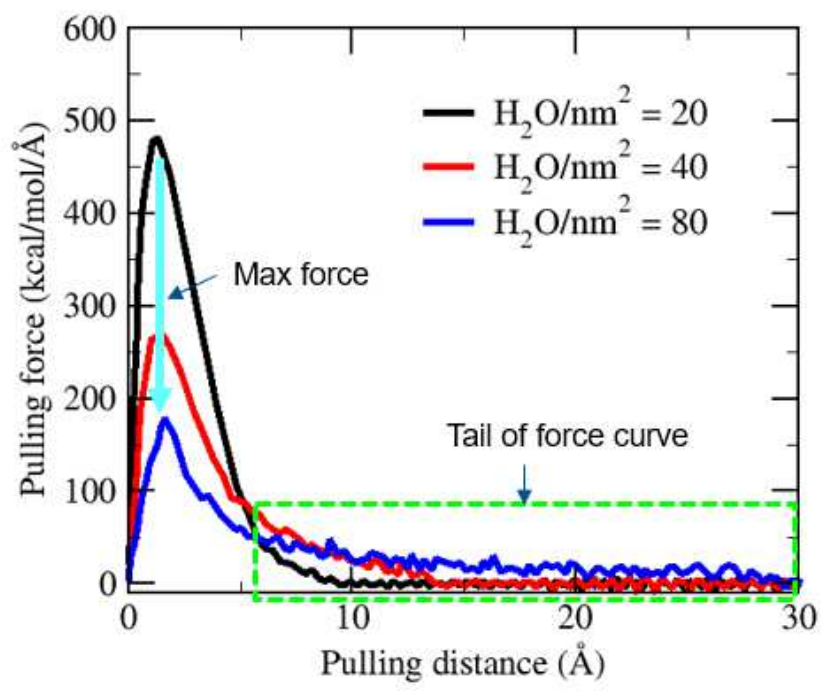

Figure 12. Change of max force and tail of force curve for increasing number of $\mathrm{H}_{2} \mathrm{O}$ during pulling test 
References

Awaja FG, M.; Kelly, G.; Fox, B; Pigram, P. J., (2009) Adhesion of Polymers Prog Polym Sci 34:948968

Berendsen HJCG, J. R.; Straatsma, T. P. (1987) The Missing Term in Effective Pair Potentials J Phys Chem 91:6269-6271

Chabria MH, S.; Smith, M. L.; Vogel, V. (Dec. 2010) Stretching Fibronectin Fibres Disrupts Binding of Bacterial Adhesins by Physically Destroying an Epitope Nat Commun 1:135

Charlier LM, K. (2012) Molecular Modeling of the Structural and Dynamical Properties of Secondary Plant Cell Walls: Influence of Lignin Chemistry J Phys Chem B 116:4163-4174

Davis DAH, A.; Yang, J.; Cremar, L. D.; Van Gough, D.; Potisek, S. L.; Ong, M. T.; Braun, P. V.; Martinez, T. J.; White, S. R.; Moore, J. S.; Sottos, N. R. (May 2009) Force-Induced Activation of Covalent Bonds in Mechano-Responsive Polymeric Materials Nature 459:68e72

Gibson LJ (2012) The Hierarchical Structure and Mechanics of Plant Materials J R Soc Interface 9:27492766

Goswami JH, E.; Fox, D. M.; Gilman, J. W.; Holmes, G. A.; Moon, R. J.; Kalaitzidou,K. (2019) The Effect of Cellulose Nanocrystal Coatings on the Glass Fiber-Epoxy Interphase Materials 12

Goyal SP, H.; Lee, S. H.; Savoy, E.; McKenzie, M. E.; Rammohan, A. R.; Mauro, J. C.; Kim, H.; Min, K.; Cho, E. (2016) Characterizing the Fundamental Adhesion of Polyimide Monomers on Crystalline and Glassy Silica Surfaces: A Molecular Dynamics Study J Phys Chem C 120:23631-23639

Heinz HL, T. -J.; Kishore, M. R.; Emami, F. S. (2013) Thermodynamically Consistent Force Fields for the Assembly of Inorganic, Organic, and Biological Nanostructures: The Interface Force Field Langmuir 29:1754-1765

Jarzynski C (1997) Nonequilibrium Equality for Free Energy Differences Phys Rev Lett 78:2690

Kargarzadeh HI, M.; Ahmad, I.; Thomas, S.; Dufresne, A. (2017) Handbook of Nanocellulose and Cellulose Nanocomposites vol 1. Wiley-VCH, Weinheim, Germany

Lacomb R (2006) Adhesion Measurement Methods Theory and Practice. CRC/Taylor \& Francis, Boca Raton, FL

Lee SHS, R. J.; Park, H.; Goyal, S.; Botu, V.; Kim, H.; Min, K.; Cho, E.; Rammohan, A. R.; Mauro, J. C. (2017) Effect of Nanoscale Roughness on Adhesion between Glassy Silica and Polyimides: A Molecular Dynamics Study J Phys Chem C 121:24648-24656

Materials Studio, Software for Technical Computation (2006), 8.0 edn. Biovia Inc., CA

Mazeau KC, L. (2012) The Molecular Basis of the Adsorption of Xylans on Cellulose Surface Cellulose 19:337-349

Min KK, Y.; Goyal, S.; Lee, S. H.; McKenzie, M. E.; Park, H.; Savoy, E.; Rammohan, A. R.; Mauro, J. C.; Kim, H. (2016) Interfacial Adhesion Behavior of Polyimides on Silica Glass: A Molecular Dynamics Study Polymer 98:1-10

Min KR, A. R.; Lee, H. S.; Shin, J.; Lee, S. H.; Goyal, S.; Park, H.; Mauro, J. C.; Stewart, R.; Botu, V.; Kim, H.; Cho, E. (2017) Computational Approaches for Investigating Interfacial Adhesion Phenomena of Polyimide on Silica Glass Sci Rep 7:10475

Min KR, A. R.; Lee, S. H.; Goyal, S.; Park, H.; Stewart, R.; He, X.; Cho, E. (2018) Grafting Functional Groups in Polymeric Binder toward Enhancing Structural Integrity of $\mathrm{Li}_{\mathrm{x}} \mathrm{SiO}_{2}$ Anode during Electrochemical Cycling J Phys Chem C 122:18190-18198

Miwa TT, R.; Numata, S. (1993) Relationship between Structure and Adhesion Properties of Aromatic Polyimides Polym (Guildf) 34:621-624

Molnar GR, D.; Martoia, F.; Dumont, J. J.; Nishiyama, Y.; Mazeau, K.; Orgeas, L. (2018) Cellulose Crystals Plastify by Localized Shear PNAS 115:7260-7265

Nishiyama Y (2009) Structure and Properties of the Cellulose Microfibril J Wood Sci 55:241-249

Paavilainen SM, J. L.; Róg, T.; Järvinen, J.; Vattulainen, I.; Ketoja, J. A. (2012) Mechanical Properties of Cellulose Nanofibrils Determined through Atomistic Molecular Dynamics Simulations Nord Pulp Pap Res J 27:282-286

Park SS, K. (2004) Calculating Potentials of Mean Force from Steered Molecular Dynamics Simulations J Chem Phys 120:5946 
Plimpton S (1995) Fast Parallel Algorithms for Short-Range Molecular Dynamics J Comput Phys 117:119

Stolper E (1982) The Speciation of Water in Silicate Melts Geochim Cosmochim Acta 46:2609-2620

Verdaguer AW, C.; Oncins, G.; Ketteler, G.; Bluhm, H.; Salmeron, M. (2007) Growth and Structure of Water on SiO2 Films on Si Investigated by Kelvin Probe Microscopy and in Situ X-ray Spectroscopies Langmuir 23:9699-9703

Yarovsky I (1997) Atomistic Simulation of Interface in Materials: Theory and Applications Aust J Phys 50:407-424

Zhang NL, S.; Xiong, L.; Hong, Y.; Chen, Y. (2015) Cellulose-Hemicellulose Interaction in Wood Secondary Cell-Wall Modeling Simul Mater Sci Eng 23

Zugenmaier P (2008) Crystalline Cellulose and Cellulose Derivatives: Characterization and Structures. Springer, Berlin

Zuravlev LT (2000) The Surface Chemistry of Amorphous Silica. Zhuravlev Model Colloids and Surfaces A: Physicochem Eng Aspects 173:1-38 
Figures
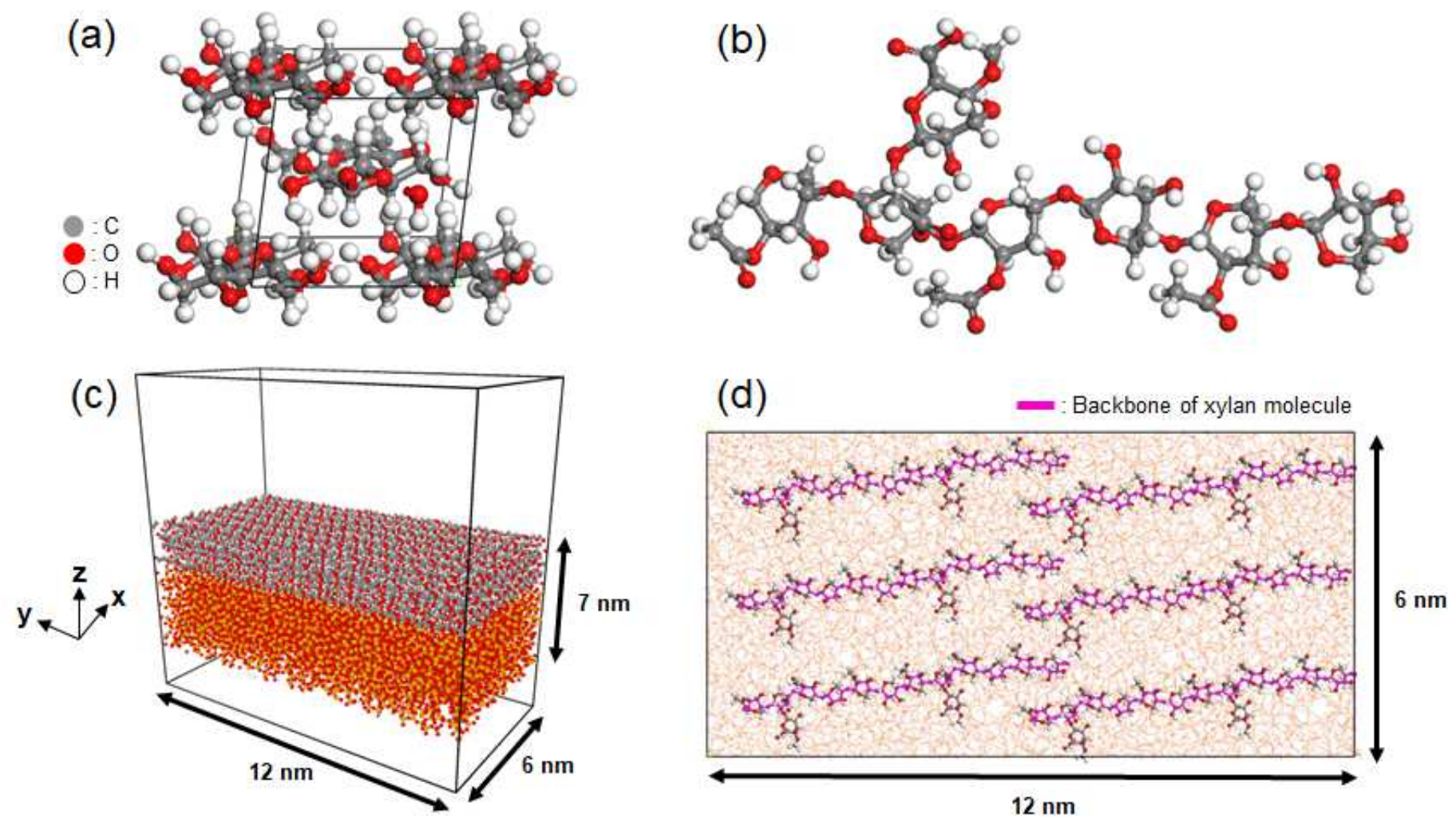

Figure 1

(a) Monoclinic crystalline structure of cellulose-I $\beta$ (b) Structure of Glucuronoxylan (xylan) hemicellulose molecule (c) Perspective view of the cellulose-silica interface after relaxation procedure (d) Bottom view of stretched configuration of 6 xylan molecules under the surface of cellulose film 


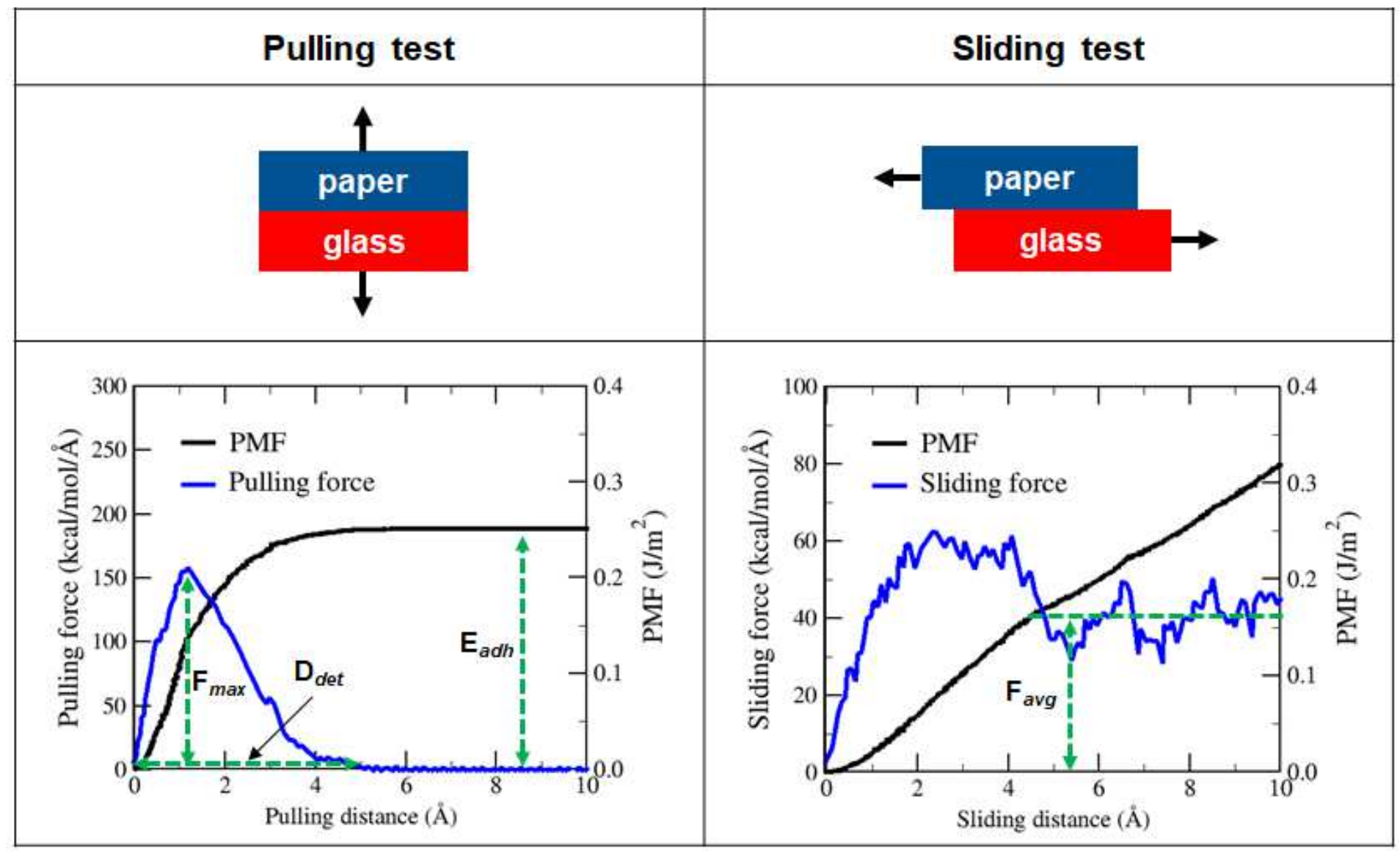

Figure 2

Comparison of pulling and sliding tests. Middle column shows basic scheme of tests, and bottom column describes typical evolution of PMF and forces and extractable adhesion properties
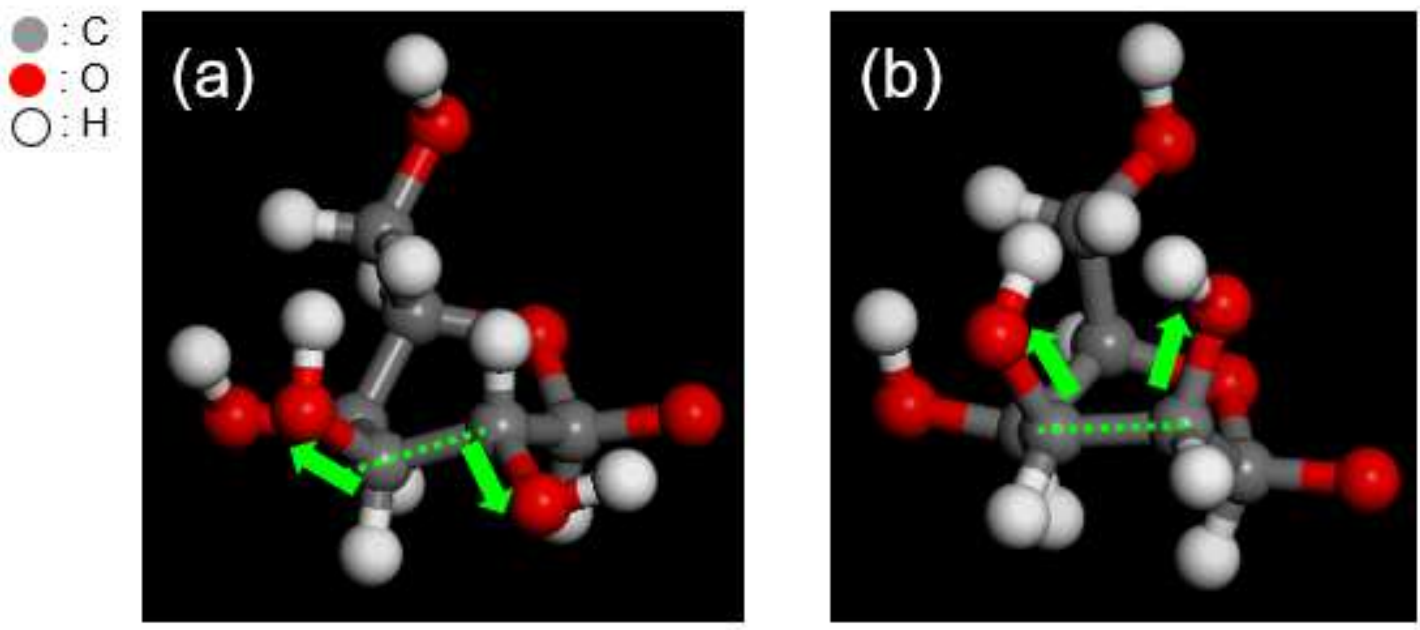

\section{Figure 3}

Chemical structures of glucose repeat units for (a) Glucan and (b) Mannan. Differences in the direction of hydroxyl groups are emphasized by green arrows. 

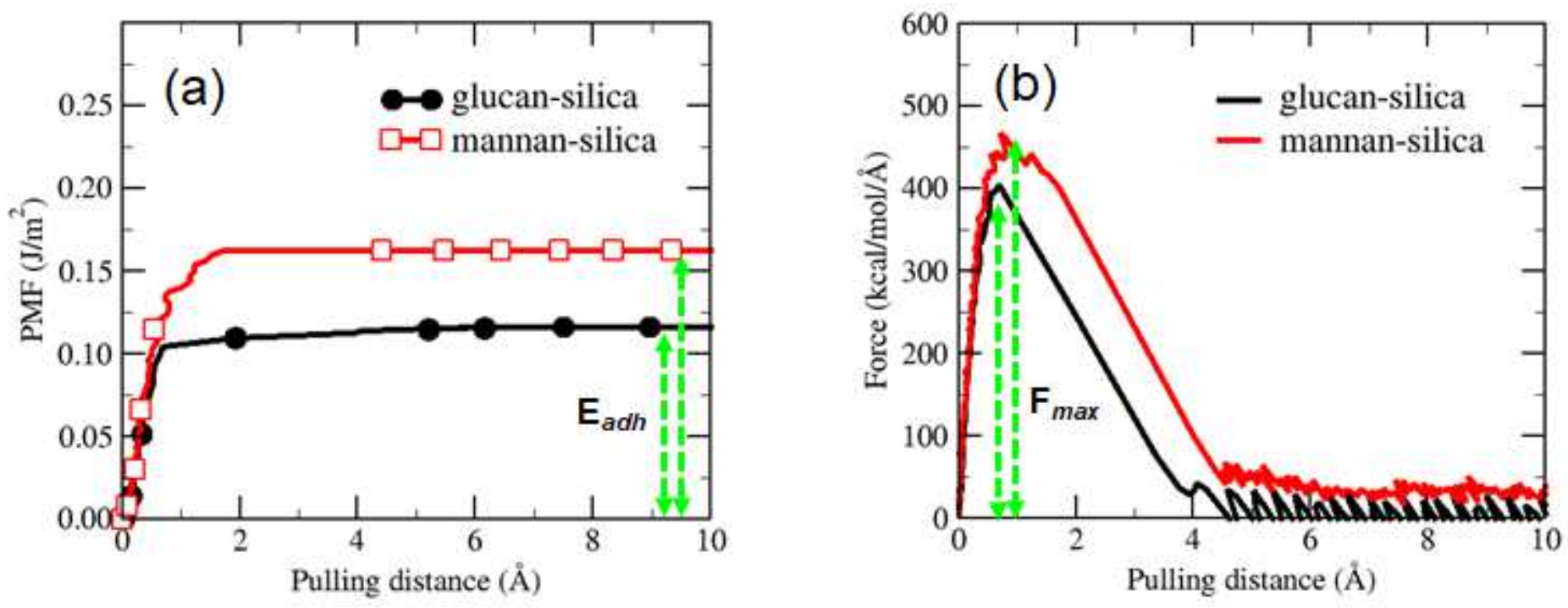

Figure 4

Comparison of adhesion properties between glucan- and mannan-silica interfaces during pulling test: (a) E_adh and (b) F_max
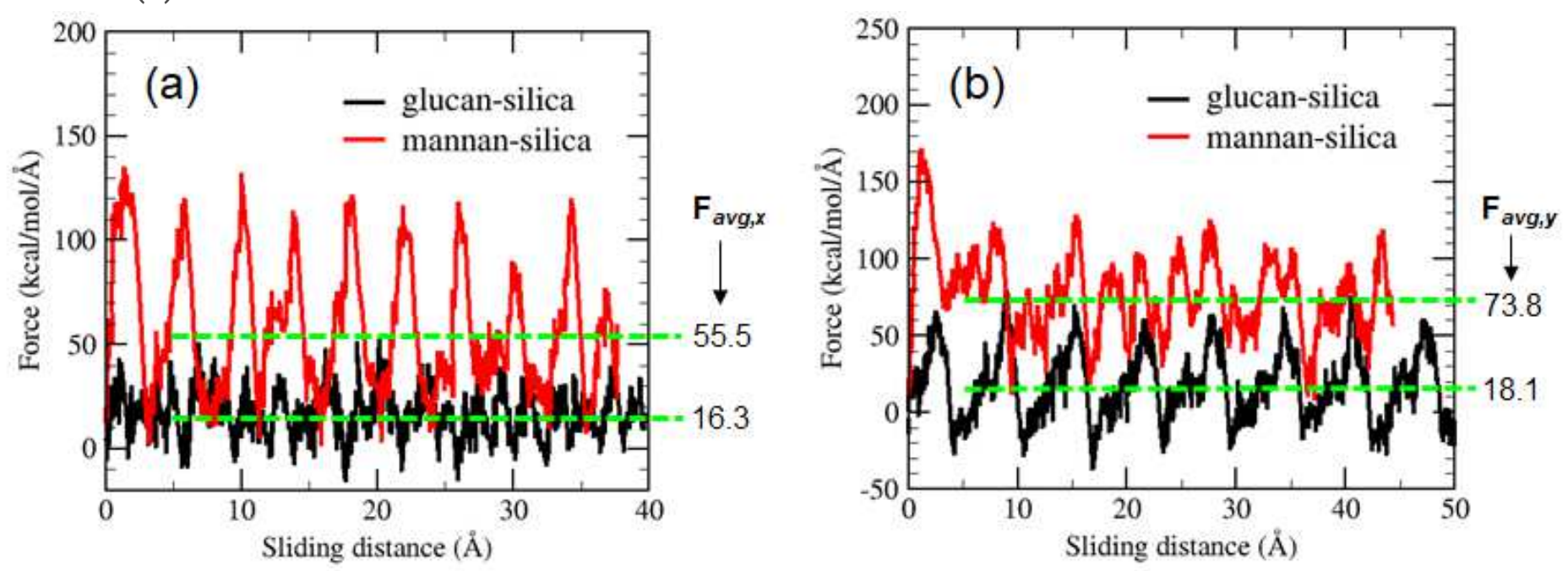

Figure 5

Comparison of sliding forces between glucan- and mannan-silica interfaces during sliding test along (a) $\mathrm{x}$ and (b) y directions. F_avg values were averaged over the sliding distance from $5 \AA$ 

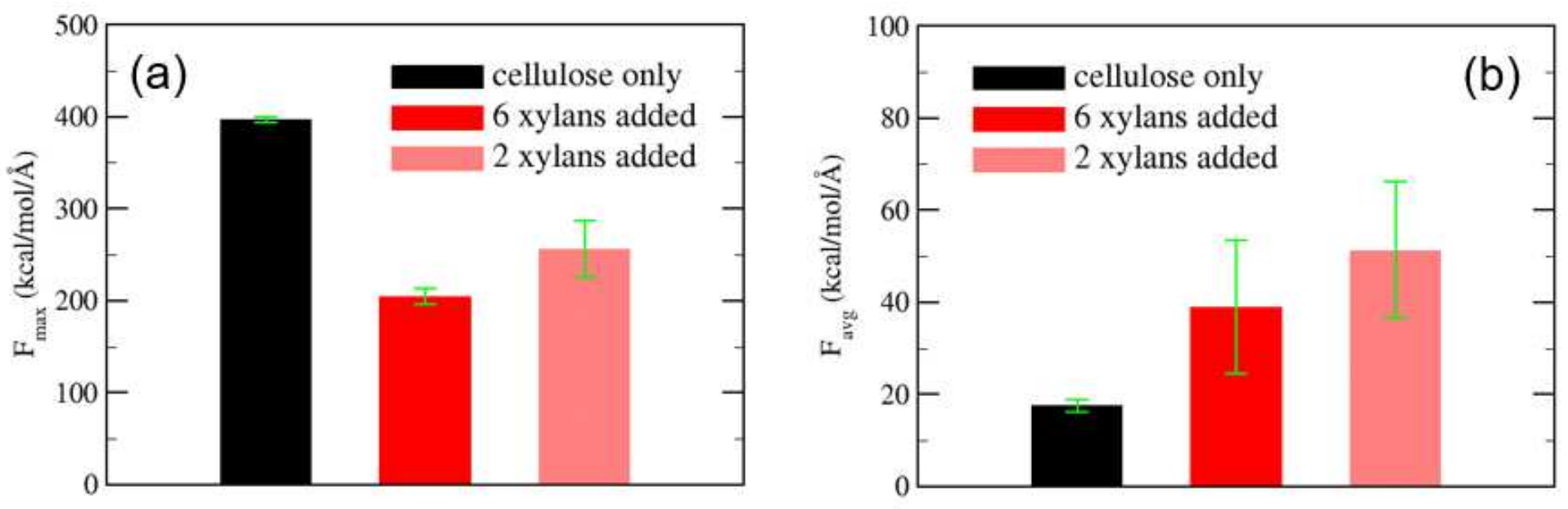

\section{Figure 6}

Comparison of adhesion forces between cellulose only case and cellulose-xylan composite case for (a) pulling and (b) sliding tests
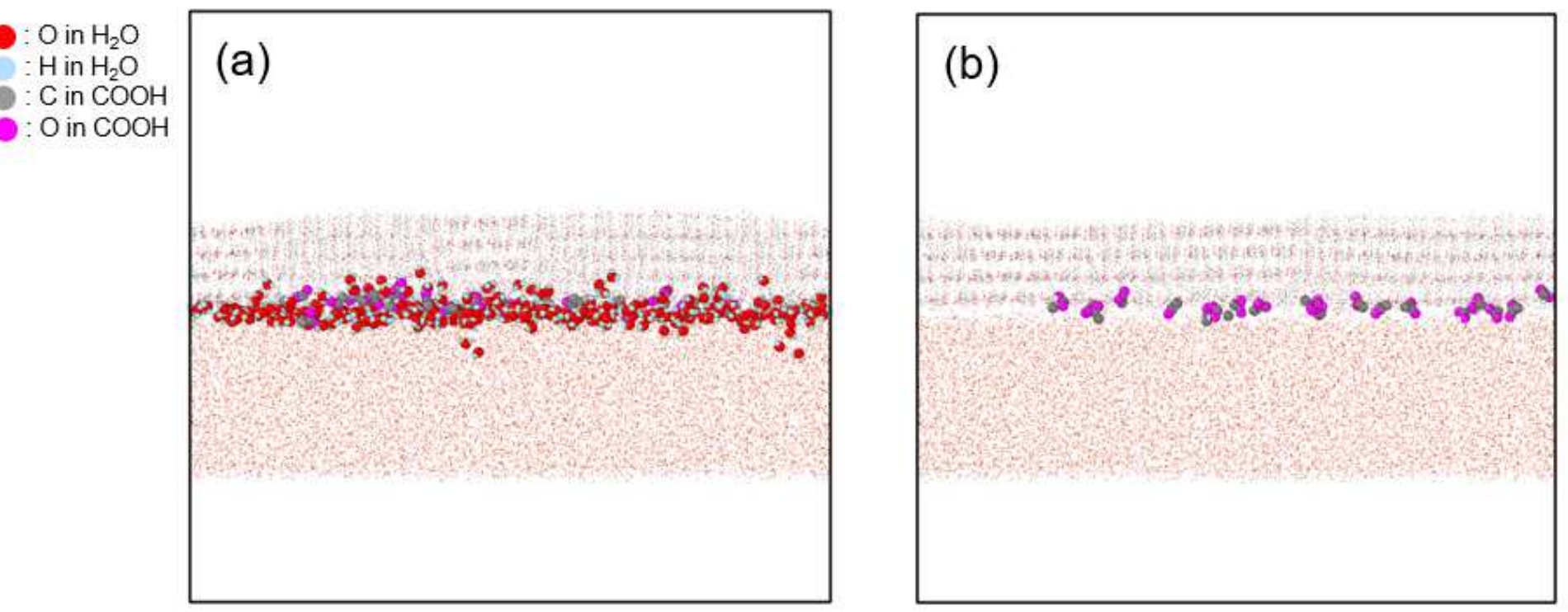

\section{Figure 7}

Snapshots of interface between cellulose-xylan composite and silica substrate under (a) $5 \mathrm{H} 2 \mathrm{O} / \mathrm{nm} 2$ and (b) dried condition. Only water molecules and $\mathrm{COOH}$ groups in xylan were emphasized with circles
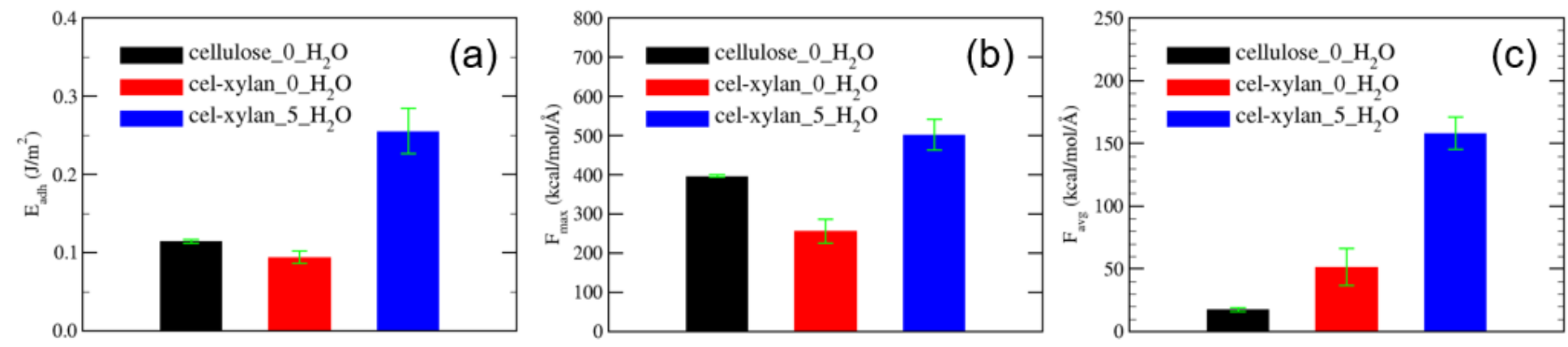

Figure 8 
Adhesion properties of cellulose-xylan composite and silica under humid and dried conditions: (a) E_adh, (b) F_max, and (c) F_avg
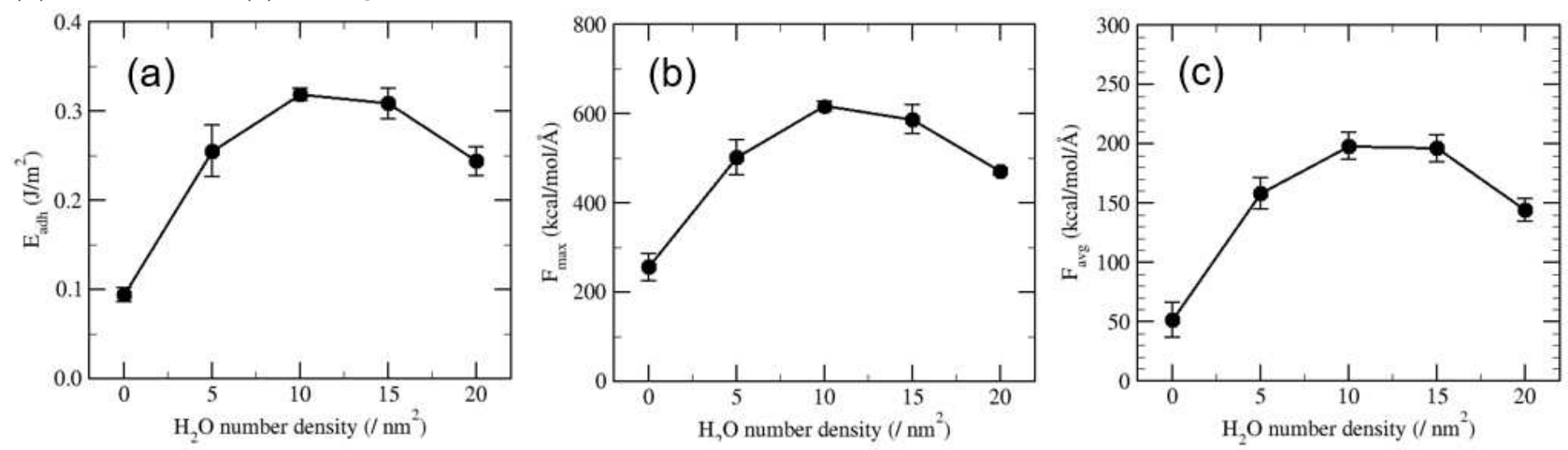

Figure 9

Trend of adhesion properties as a functions of $\mathrm{H} 2 \mathrm{O}$ number density: (a) E_adh, (b) F_max, and (c) F_avg

: Oxygen in water

: Hydrogen in water

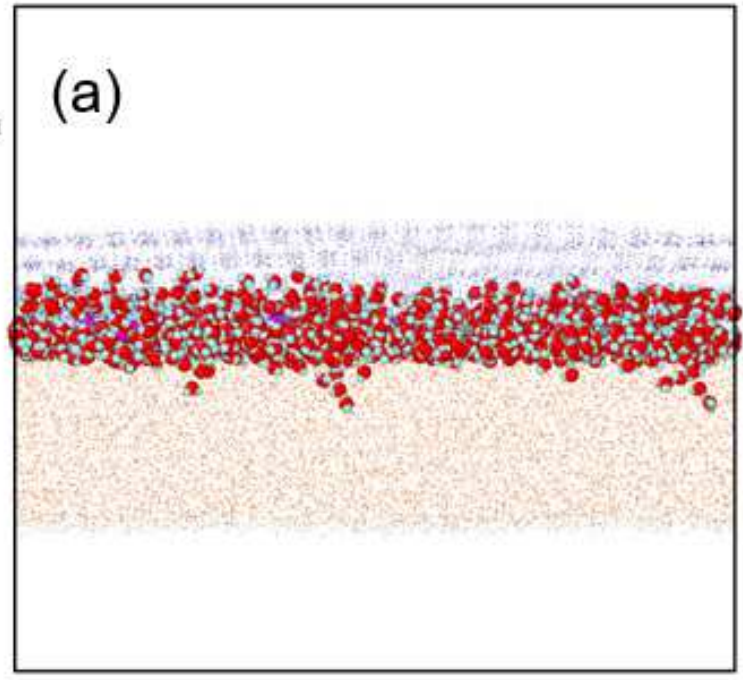

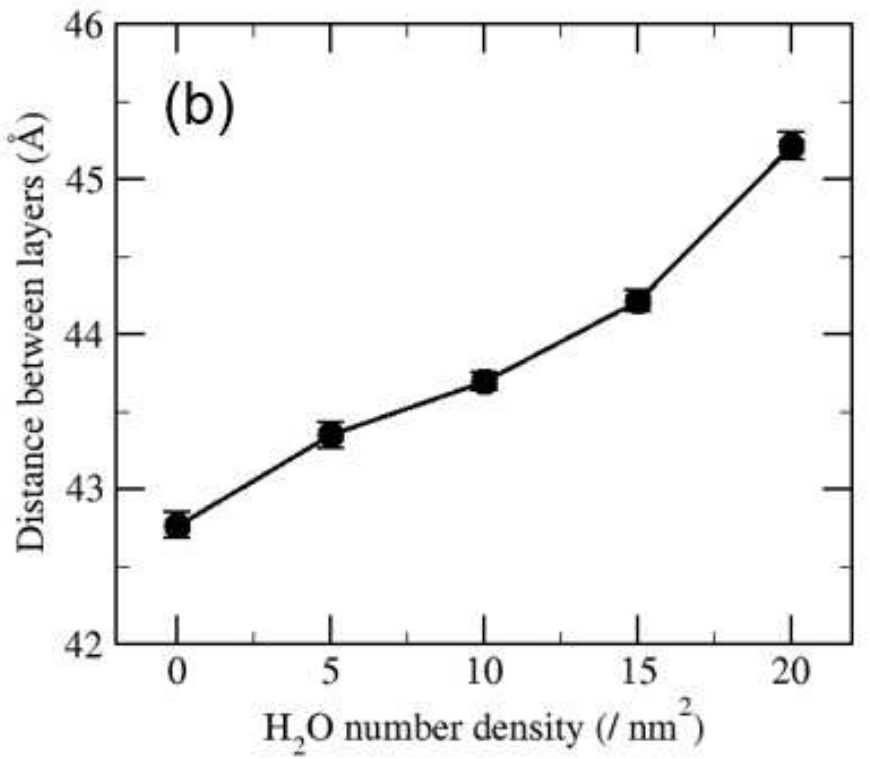

Figure 10

(a) water layer between cellulose film and silica surface at $20 \mathrm{H} 2 \mathrm{O} / \mathrm{nm} 2$ (b) Distance between cellulose and silica increases with increasing humidity, which implies molecular interactions between the layers are reduced 

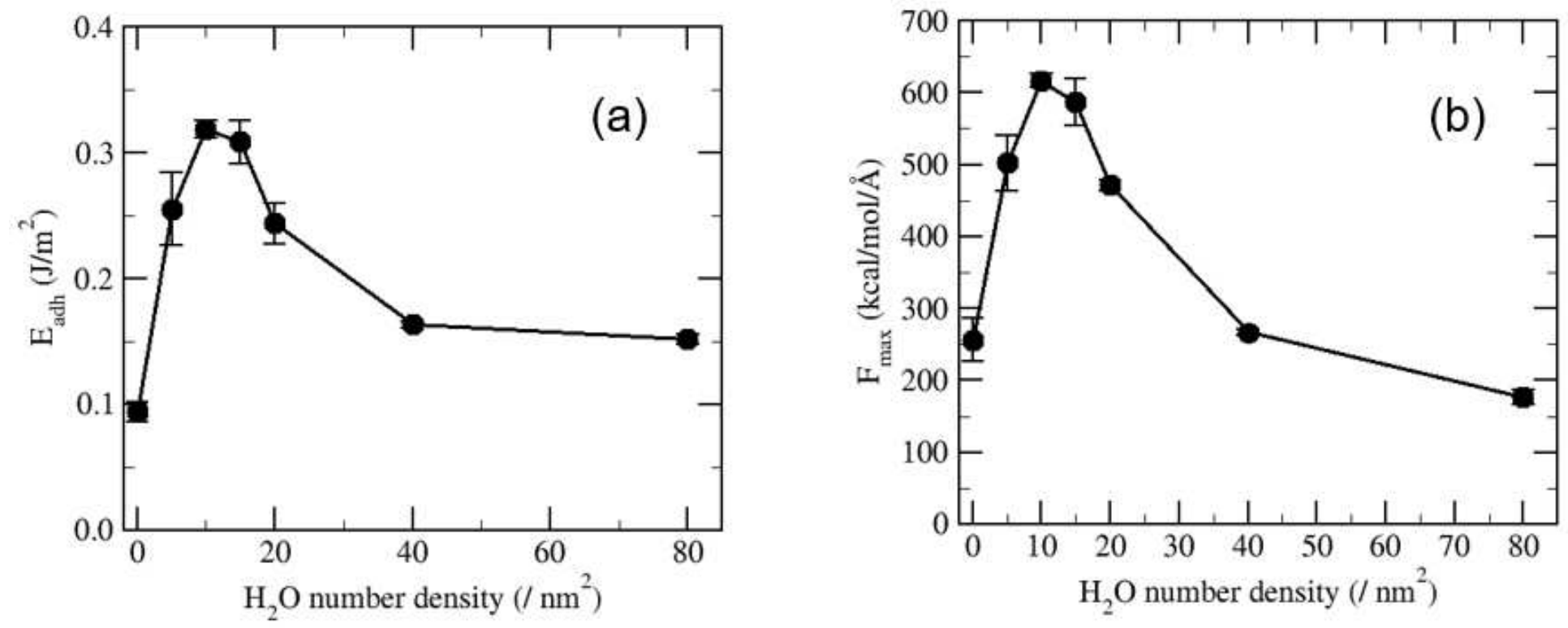

Figure 11

Curves of (a) E_adh and (b) F_max for paper-glass interface extended range of $\mathrm{H} 2 \mathrm{O}$ number density up to $80 \mathrm{H} 2 \mathrm{O} / \mathrm{nm} 2$

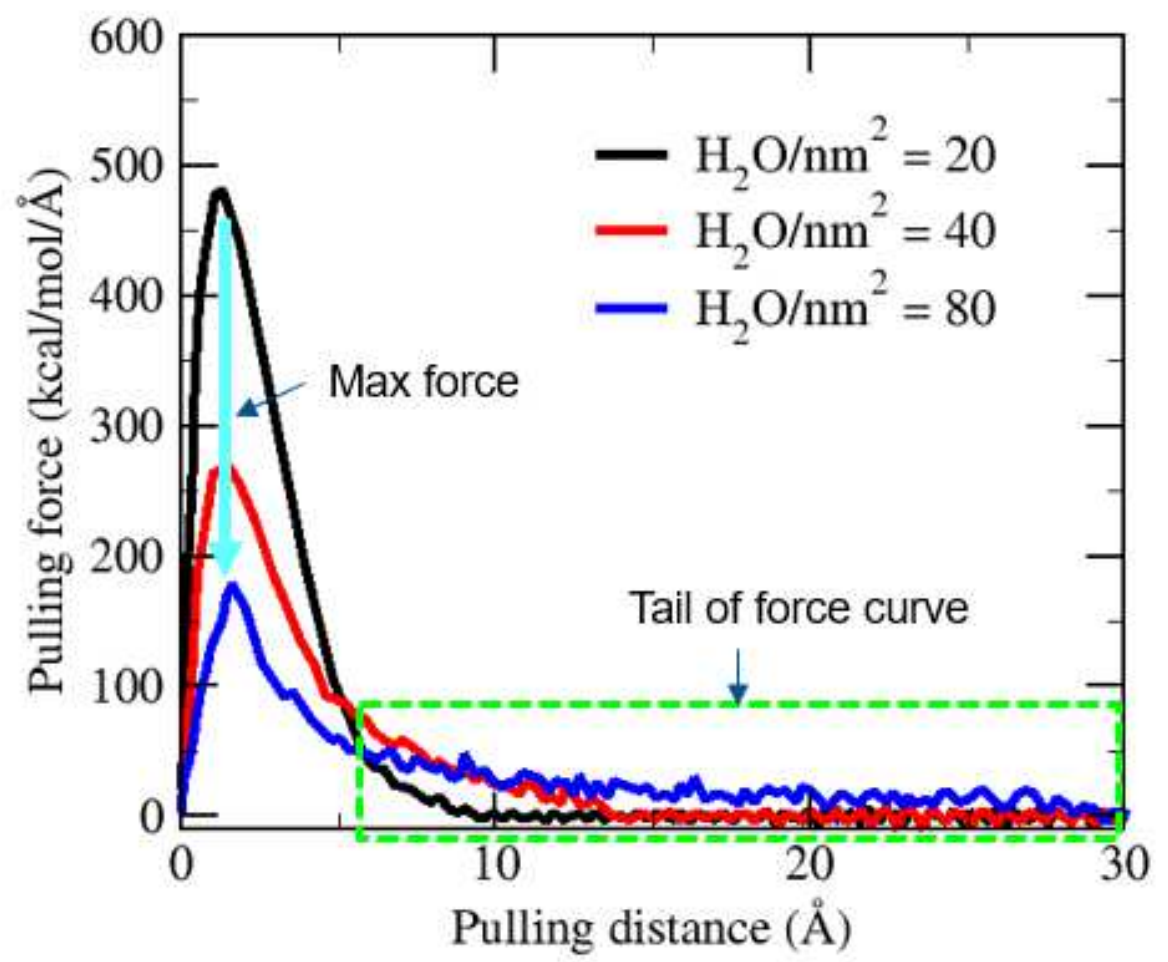

Figure 12

Change of max force and tail of force curve for increasing number of $\mathrm{H} 2 \mathrm{O}$ during pulling test 